\title{
A Reversed Construction between Deities and Demons: A Case Study of Ne Zha: I'm the Destiny
}

\section{Hai Tang* Yang You Mengchun Ma}

School of Foreign Languages, Wuxi Taihu University, Wuxi, Jiangsu, 214000, China

\section{ARTICLE INFO}

Article history

Received: 5 February 2021

Revised: 12 February 2021

Accepted: 9 April 2021

Published Online: 16 April 2021

Keywords:

Ne Zha: I'm the Destiny

Animation

Chinese school

Gods and demons

Hero

\begin{abstract}
In 2019, Ne Zha: I'm the Destiny won more than 5-billion-yuan box office in Chinese movie market. Although the box office is not the only criterion for judging the quality of an animated movie, Ne Zha breaks a certain stereotype in storytelling, technical means, and the setting of the positive and negative characters. This potentially indicates that in producing an animated film, Chinese artists start to pay more attention to how to refine a movie production, or how to make the story more interesting. One of the attractive elements of this movie is that, Ne Zha has made a breakthrough in creation of a character of Children's hero, and is totally different from its previous versions: with his joy and sorrow, audiences see the characteristics of Ne Zha as a nice as well as an aggressive boy. This partially because in setting the role of $\mathrm{Ne} Z \mathrm{Zha}$, boundaries between the good and the evil or between beauty and ugly are not so clear in the animation, while it features that Chinese cartoons are set to focus more on authenticity rather than their instructive functions. Additionally, the change of the image of $\mathrm{Ne} Z \mathrm{Zha}$ and the subversive characters re-created in the movie have metaphorical significances, featuring how "Chinese school" reconstructs the subjectivity of Chinese stories in contemporary world.
\end{abstract}

the originality (Lv Xinyang, 2020; Qin Xiaolin, 2019; Sun Lijia, 2020; Zhang Jin, 2019), even though they are in relation to a shifted role of the character or the adaptation of the story.

Taking Ne Zha: I'm the destiny, when this animation came out in 2019, an extensive discussion was held immediately in academic field, rethinking the function of "Chinese School" in movie studies (Chen Guannan, 2019; Ding Yaping, 2019; Hu Yihao, 2019; Pan Jian, 2020). What the movie argued is about the choice of making of the character of $\mathrm{Ne} Z \mathrm{Zha}$, whether it should focus on inher-

*Corresponding Author:

Hai Tang,

Associate Professor, PhD in Media and Cultural Studies;

Corresponding address:

68 Qianrong Road, Binhu District, School of Foreign Languages, Wuxi Taihu University, Wuxi, Jiangsu, 214000, China;

E-mail: carlatube@qq.com. 
iting a traditional children's hero, or it can relate to an anti-tradition subversion? So far as we can see from the $\mathrm{Ne}$ Zha, it has broken a Chinese-based creative experience, newly constructing the Chinese myths by shifting the relation in between the two "magics": the deity and the demon, with realistic meanings.

In other words, either in its artistic style or in its narrative setting, the adaptation of Ne Zha has not 'divorced' from the style of "Chinese School". The animation of the $\mathrm{Ne}$ Zha comes from a traditional Chinese mythical story, while it differs from its previous works in that it unfolds in a relatively confrontational storyline. To rephrase, the character of Ne Zha is a mixture of the good and the evil. This might form the primary elements of the subversion in the animation, while it in turn gives an initial change to "Chinese School" for practicing the "telling Chinese stories" in the context of globalization (Chen Guannan, 2019: 132; Ding Yaping, 2019:62).

What does the new image of Ne Zha look like in its 2019 animation movie? Why and how does it reflect the new perspective of the "Chinese School"? These are the questions raised in the paper and in the following sections, it is going to lift the veil through in-depth elaboration, analyses, and deconstruction of the character of this animation film. That is to say, the second section describes the relationship between animation and Chinese mythology. The purpose, however, is to argue that mythological stories may lead to the worship of the god; the image and deification of heroes created by the Chinese School in movies are typical examples. The third part of the paper analyses of the characters of the movie. It points out that when talking about main character or the supporting ones in the movie, one thing in common is that they all interact with the term 'subversion'. Next section talks about the new narrative way of the "Chinese School" in making Chinese animation. By getting rid of the influence of the "invasion of Western culture", Chinese filmmakers gradually win the market. The idea is particularly emphasized in the conclusion part, meaning, the fundamental way to develop Chinese animation is to tell real Chinese story to the world.

\section{Chinese School and the Traditional Writing about Mythical Heroes in Chinese Animation Films}

From 1950s to 1980s, China can be said to form its unique artistic styles in the history of the animation industry, and the "Chinese School" is a summary of those styles created by Chinese animated artists during that period. In 1960s, a series of ink animations were produced,
Xiao Kedou Zhao Mama (Tadpoles, see Figure 1), for instance, was especially influential. Later, the work $D a$ Nao Tiankong (Uproar in Heaven, see Figure 2) shifted the ink model to the focus of the narration of a story, and made great success. Foreign media commented that it has a similar style as Disney works, but it tells a great Chinese story to the world (Ding Yaping, 2019:59).

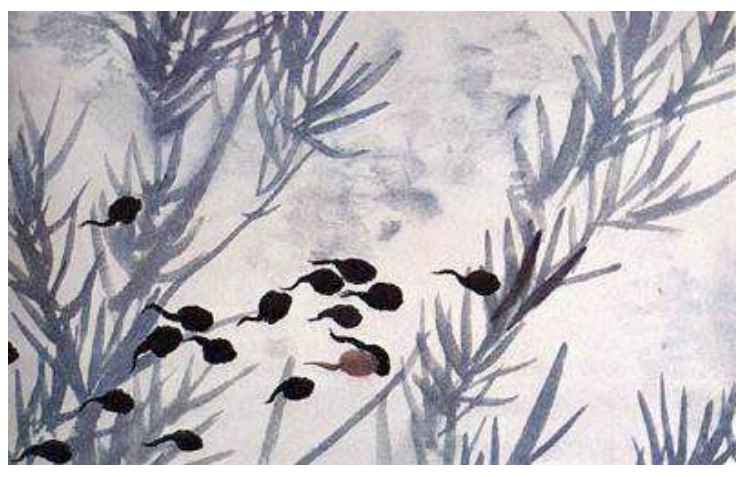

Figure 1. Tadpoles

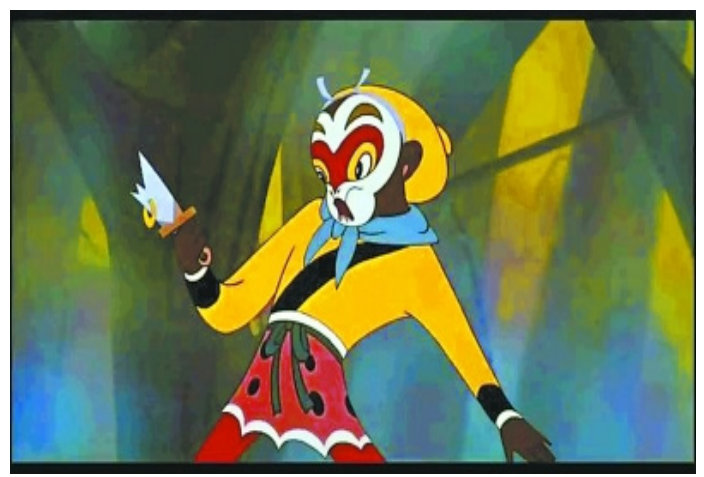

Figure 2. Uproar in the Heaven

Source: https://image.baidu.com

Compared with animated films in the West and other regions, Chinese animated films appeared belatedly, with a lower level of technology and other problems. This is partially the reason China focused on developing its story and embodying of nationality in making the films. As Ding Yaping argued:

Chinese animation has won the awards in competitions, and of course, it has been recognized by the international film industry, but it is still lagging behind the works in theory and overall concepts. Animation enthusiasts may have their own Miyazaki or Disney roles but not a Chinese name. The former two symbols have a strong influence on both audiences and the animation market in the world. (2019: 60)

Although the writing styles in both Chinese and English are not the same, myths and legends have been the two important factors in coining animation movies. Yuan Ke (2016:4) noted that the creation of the deity in China 
comes from a particular system: religiosity. Taoism and Buddhism, for instance, are the two largest god-making elements; the latter spontaneously leads a hero to its worship in Chinese history.

In addition, the intervention of a hierarchical system is a supplement to form folk arts. The heroes in Chinese mythological films were often big names, familiarized by the public with a high degree of an identity of nationalist or at least good persons (Lv Xinyang, 2020; Sun Lijia, 2020). Sun Wukong, as we all know, was designed in Uproar in the Heaven as a heroic image of "brave, brilliant, powerful and wise". While demons are evil, they will be the loser at the end of the story (see Figure 3). Thus, early animation works in the grand narrative style, by Chinese myth and legacy constructed the relationship between the gods and demons, were particularly clear. It has become a cultural symbol in Chinese films.
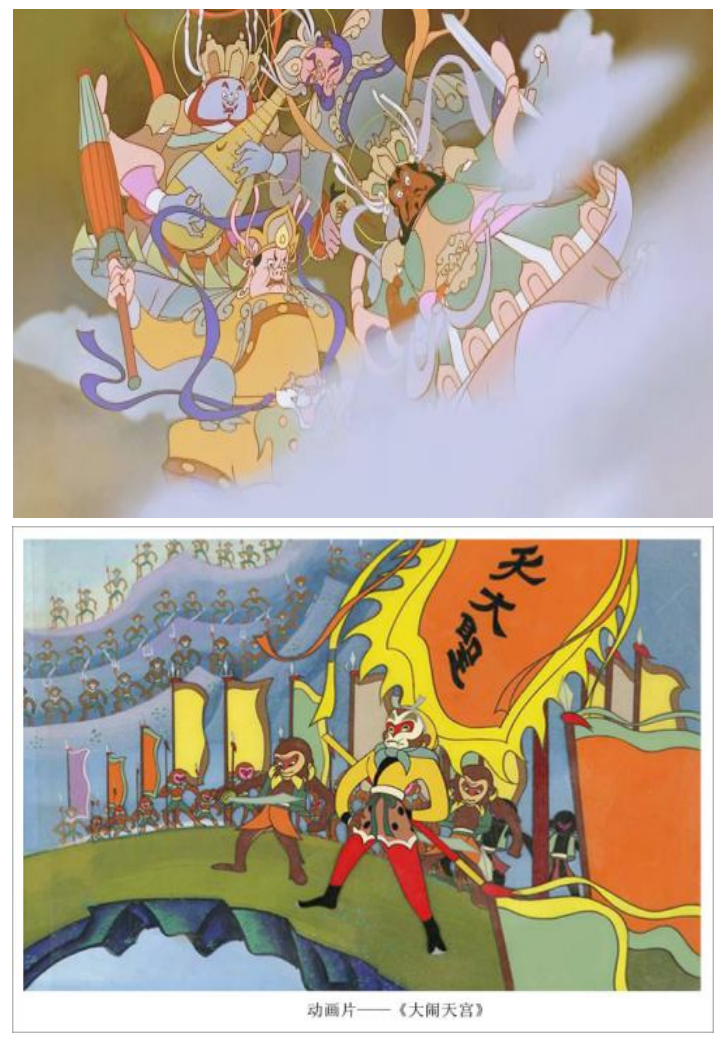

Figure 3. Uproar in the Heaven

Source: https://image.baidu.com

As there has been an all-round transformation in China's economic and cultural areas after late-1980s, movies possibly became less instructive but more entertaining. An introduction of Hong Kong films in the 1990s, especially its post-modernist style in movie industry impressed a lot of filmmakers. Zhou Xingchi, his ridiculous comedy reconstructed a traditional myth and mythical hero Sun Wu- kong, which were learned and adapted in later on Hong Kong films. Sun Wukong in a series of Zhou's The Journey to the West was irrational, poor and easily annoyed (see Figure 4), it subverted a traditional image, while making Zhou Xingchi as a representative in Hong Kong.

To simplify classic contexts, to normalize heroes, to reconstruct elitism, and to satirize traditional values, etc. in every way, the Hong Kong version The Journey to the West carried a sense of Hong Kong humor, derived from the culture of Hong Kong. (Sun Lijia, 2020:49)
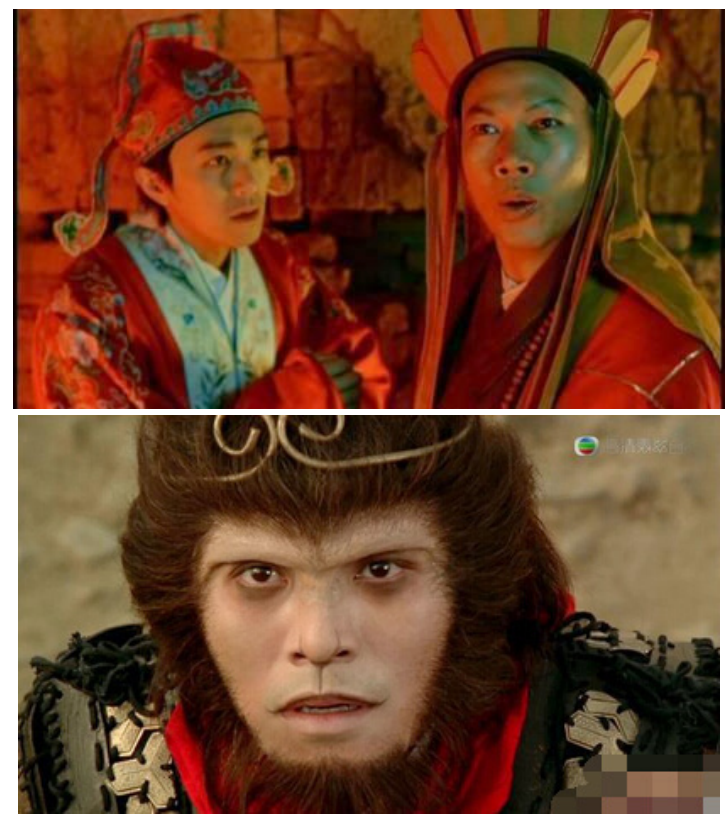

Figure 4. Journey to the West (directed by Zhou Xingchi) Source: https://image.baidu.com

"Chinese School" shows differences in both artistic and market fields in different periods. Early animations focused more on the narration, while at the same time, they aimed to emphasize an instructive function in the story. Filmmaking contains lots of preparations; the needs to face the challenge of the market, and the needs to satisfy the audiences shall be included as well. If Zhou Xingchi's success is based on his skillfully using his anti-tradition to shape a grassroots hero, then Ne Zha: I am the Destiny structured an anti-tradition as well, while the subversion lied on its reconstruction of the relation between the gods and the demons.

\section{Characteristics of the Mythical Hero in Re- versed Constructions:The Case of Ne Zha: I'm the Destiny}

As mentioned in the previous section, Chinese traditional culture is deeply influenced by Confucianism, the mythological animation works and the construction of 
"heroism" have a strong sense of power. In other words, the myth of a hero is built to defeat demons and monsters, this partially because the main audiences of the movie are children and young people; the victory of justice, to a certain extent, may play an instructive role. For example, in Ne Zha Nao Hai (Ne Zha Conquers the Dragon King, 1979), Ne Zha was designed as a hero who sacrificed his life to protect people (see Figure 5). Such a character was set on a moral level that a division between "good" and "bad" or "evil" was seen as an intuitive relation between gods and demons.

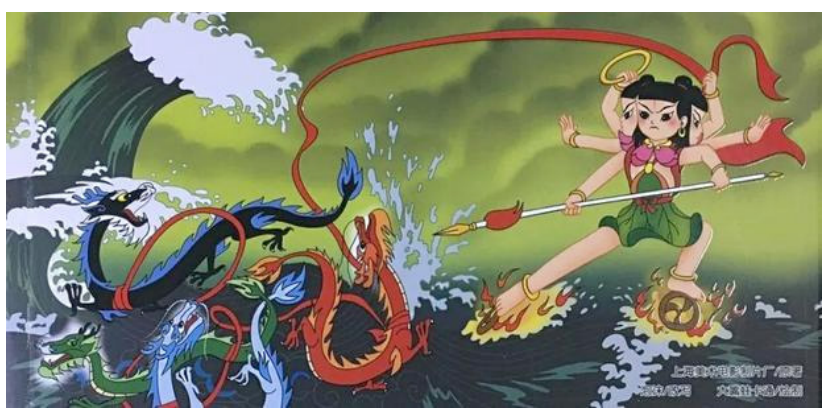

Figure 5. Ne Zha Conquers the Dragon King

Source: https://image.baidu.com

There is also a certain continuity in shaping of the image of Ne Zha in its previous animations. Before the Northern Song Dynasty, Ne Zha was shaped as "Seeing with an Evil's Eye." An earlier book recorded Ne Zha in Yuan Dynasty and wrote that "viewing from three-head, and waving by six-arm." In the Journey to the West, Ne Zha was described as a little kid, "looks strange but is very strong." A detailed description of Ne Zha was in the Story of Chinese Gods, "his feet are on the fire, with a sharp gun in his hands." (Liu Wengang, 2009). Meanwhile, the ring, the slug and the gold brick appeared not only as three magic weapons, but also as a representative image of Ne Zha.

In the 2019 animation film, such an iconic image of $\mathrm{Ne}$ Zha hasn't changed that much, while filmmakers focused more on how to tell an old story with new ideas. That is, to tell social stories, and to allow audiences to be engaged, interested and able to understand and act on the content. Generally, the content in this paper is called anti-tradition.

The first viewpoint of anti-tradition in Ne Zha: I'm the Destiny is that, Ne Zha was reversely constructed by his birth story. For instance, the bead set in the movie is not the one as its saint originality but featured with demonic function, resulted from a mistake made by Tai Yi and thus, transferred Ne Zha to be a demonic baby, carrying a rebellious personality (see Figure 6 and 7). It is opposed to an orthodox heroic image, who was lovely, rational, and wise.

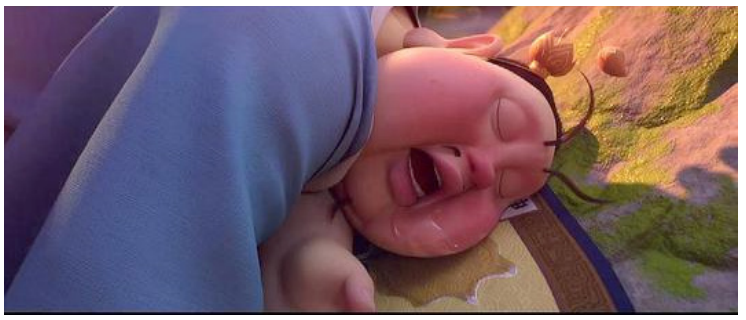

Figure 6. Taiyi in Ne Zha: I'm the Destiny

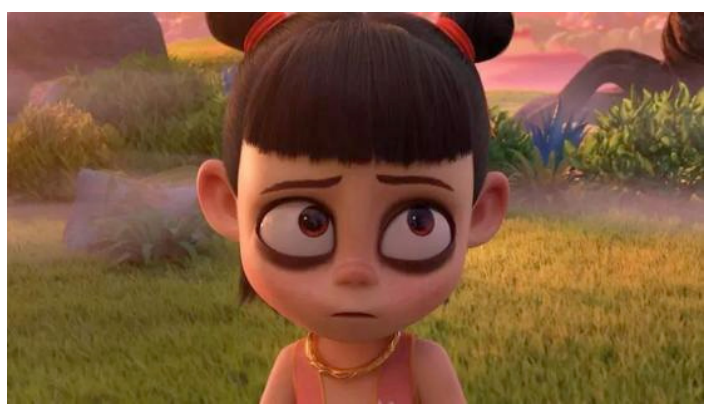

Figure 7. The Image of $\mathrm{Ne} \mathrm{Zha}$

Source: https://image.baidu.com

The second viewpoint of anti-tradition in the animation is that, the use of rewriting the death of $\mathrm{Ne} Z \mathrm{Zha}$ constructed a subversion of tragic fate. Looking back at this mythical hero, Ne Zha is indeed a tragic one, while the animation used "Changing your life and finding your destiny" as a new theme (Ding Yu, 2020:89), concentrating on the needs of love in a family (see Figure 8). Under such a reversed construction, the hero brings an immediate emotional resonance from nowadays audiences.

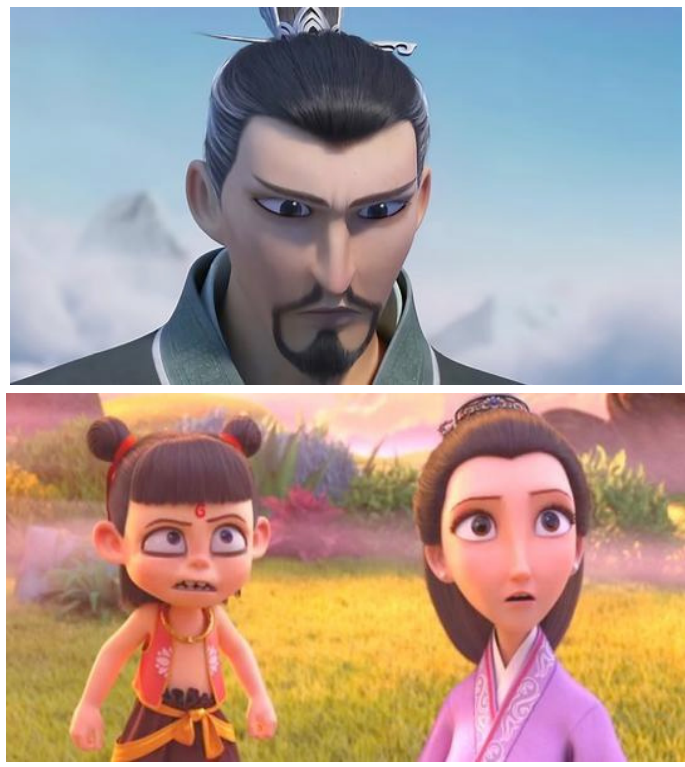

Figure 8. Ne Zha and his Parents in Ne Zha: I'm the Destiny

Source: https://image.baidu.com 
As mentioned above, deity and magic are two sources of creating animated characters, though, an old version of mythology being likely to clarify the relationship between gods and demons was not given a clear subdivision in this film. The bead was attached to Ao Bing at his birth, to some extent, it was the bead that forced Ne Zha to share a similar demonic identity with Ao Bing (see Figure 9). Such a reversed construction of the deity and the demon can no longer be said in a word of good vs. bad, or right vs. wrong, as each character has its internal logic and behavioral pattern.

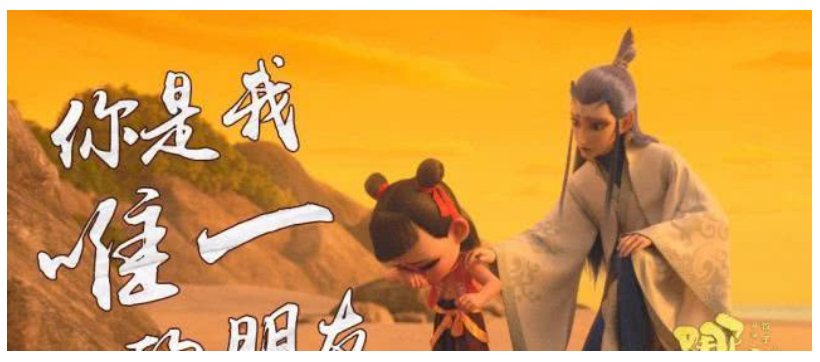

Figure 9. Ne Zha and Ao Bing in Ne Zha: I'm the Destiny

Source: https://image.baidu.com

The biggest changing part of the narrative style of $\mathrm{Ne}$ Zha: I'm the Destiny, as can be seen, is that it starts to use classic myth or fable to shape the real role of a children's hero, and to differentiate it from its previous animation works. The outline of the narration can be grouped in Ne Zha's family, his determination of friendship, and his self-awakening (Lv Xinyang, 2020:128). It is this clear and reasonable logic that making the animation conceive new sense of meaning. Meanwhile, Ne Zha is no longer a hero, but converging good and bad behaviors and characteristics; such a deconstruction of the character of Ne Zha, along with his cynical but strong personalities have won lots of audiences.

\section{Subverted Characters Built in Ne Zha: I'm the Destiny in the View of New Chinese School}

What have been said in the last section is about the anti-tradition factors in Ne Zha: I'm the Destiny. Such anti-tradition has shifted the character of a hero, being good or a great person only, toward a person with certain potentiality and possibility of being bad or an evil. In other words, heroes are also human beings, they can be positive but also have a negative side; they might be betrayed, and isolated. Probably the combination of current social reality with a traditional myth story is one of the reasons and explains why this animation won box office in 2019 . $\mathrm{Ne} Z \mathrm{Zha}$ is not the only one indeed, other animated films such as Monkey King: Hero is Back (2015), Big Fish and Begonia (2016), and the Happy Sheep and Grey Wolf series have also achieved the box office in recent years (Hu Yihao, 2019; Li Junhua, 2019; Qin Xiaolin, 2019). It is arguably to say that integrating social contents into animation stories is new but is a good try practiced by the "Chinese School".

Taking Ne Zha: I'm the Destiny, first, the main character created in previous animation works was a kid who is a 'lack of love', and aggressive. The main theme of the movies was in struggle with the "filial piety" and strict barriers to a hierarchical system in Chinese old times. While Ne Zha in its new version is the one who is ugly as well subversive, but is loved by his parents. Such a story setting is in line with the perception of a "Happy Family" in contemporary society (Chen Guannan, 2019:133), reconstructing Ne Zha to cater to a narrative structure of the mainstream theme.

Second, the animation highlighting the significance of individualism has greatly attracted young people. Besides $\mathrm{Ne}$ Zha, the characters of the supporting role, Ao Bing, and Shengong Bao, for example, they also are in struggle with their birth and destiny. Take Ao Bing, who was born as a demon but who is set as an absolute hope to change the fate of his dragon family. The unique relation between the deity and demon implies a projection of the hard-working young people in current society who are struggling with their life and destiny, synchronizing an emotion of the working-class audiences, their needs of self-actualization.

Third, Ne Zha, in its animated version, showed a high degree of nationalism (Ding Yaping, 2019:61). By telling about how Ne Zha was born, how he grows up and becomes a friend of Ao Bing, and why they fight for the fate, this animated work accurately grasped audiences' attractions, from where they understand Ne Zha's grievance, anger and rebellion. Such an empathy can be seen as one of the successes in making the film, that a reversed main character connects with China and its motivation to change in fate becomes reasonable.

Overall, the animated image of the main character and supporting characters in Ne Zha: I'm the Destiny subverted their old images or the magic of the god, giving the audiences a richer imagination and greater joy. From watching the movie, audiences can see that Ne Zha is no longer the one as its originality as children's hero, but is combining of saint with human nature, while at the same time, the change may have a deep or lasting impact on their own lives (Chen Xu and Wu Yangyang, 2020:199200). In other words, the revised characters designed in this animation work is not only the aesthetic changes they 
shown to audiences, but they also represent how Chinese animation filmmakers to explore the path to telling real Chinese stories in their artistic works to the world.

\section{Conclusion}

To rephrase, in the animated film Ne Zha: I'm the Destiny, a reversed construction of the "deity-demon" relation is the biggest challenge, confronting a legislative rule between the good and the evil, satirizing an existing prejudice that demon becomes deity could be an over-lasting consequence. In other words, the animation film explains what the term "Destiny" is; through a reversed as well as an converged relation between gods and demons to set positive and negative characters. The main character $\mathrm{Ne}$ Zha, for instance, who was born as similar as a monster, carried an identity of the 'evil'. While Ne Zha has been in struggle with the prejudice, harm and intolerance from outside, the purpose is to seek a further demonstration of self-identification.

In addition, the re-constructed images have filled the narrative loopholes of making Chinese stories, starting with small changes, as shown in Ne Zha's dark circles, Ao Bing's long horns, along with a fat Tai Yi, and a stuttered Shengong Bao. Finding the beauty from the ugly one has supported an anti-profiling characteristic. The aesthetic effect of the film, along with its metaphorical expression of the theme "I'm the Destiny" are in line with the appreciation habits of contemporary audiences. Either Ne Zha, Ao Bing or Shengong Bao, their fighting against their birth, destiny or prejudice imply how young people relentlessly pursue their dreams; they in turn correspond to the mainstream theme of building a positive socio-cultural atmosphere in China.

Furthermore, the use of red, yellow, and black colors in this animated film also has a special meaning. Ne Zha standing in the fire stated his determination to fight against his fate, such an adaptation won't be a simple conforming to a psychological need of the audiences, it may imply that China have done to make efforts and to construct its future. This indeed functions a critical thinking with the new style of the "Chinese School". From a previous technical imitation of the West to its independently producing the work, Chinese artists are moving towards autonomy and creativity to showcase their animated talents. It can be said that their efforts to "tell Chinese stories" is becoming a growing consensus, enhancing the view of national symbols on their animated works to communicate with the world.

\section{References}

[1] Chen, G. N., (2019). On Discussion of the Aesthetic dimensions of Ne Zha: I'm the Destiny (in Chinese). Movie Literature, 23, pp.131-133.

[2] Chen, X. and Wu, Y. Y. (2020). Deconstruction of the Concept of Destiny in Ne Zha: I'm the Destiny (in Chinese). Journal of Changchun Normal University, 7:197-200.

[3] Ding, Y. (2020). Analyses of the Similarities and Difference of the Characteristics of Ne Zha in Ne Zha: I'm the Destiny and The Story of Chinese Gods. Radio and TV Journal, 9, pp.89-90.

[4] Ding, Y. P. (2019). The Past, Present and Future of the Chinese School and the Chinese Animations (in Chinese). Yuehaifeng, 2, pp.55-63.

[5] Hu, Y. H. (2019). Research on Changes in Building Nationalism in Domestic Animations (in Chinese). Movie Review, 19: 47-52.

[6] Li, J. H. (2019) The Expression of Chinese Styles in Domestic Chinese Films (in Chinese). Research on Digital Media, 36(11), pp.51-53.

[7] Liu. W. G. (2009). On Discussion and Evaluation of the Image of Ne Zha (in Chinese). Religious Studies, 3, pp.178-183.

[8] Lv, X. Y. (2020). Cultural Deconstruction and Remodeling of Ne Zha in Ne Zha: I'm the Destiny (in Chinese). Movie Literature, 4, pp.127-129.

[9] Pan, J. (2020). An Observation and Analysis on 2019 Chinese Animation Films (in Chinese). Contemporary Cinema, 2, pp.27-34.

[10] Qin, X. L. (2019). From a Legend towards a Human: Rethinking about the Changing Images of Sun $\mathrm{Wu}$ kong (in Chinese). Movie Review, 16:84-7.

[11] Sun, L. J. (2020). Cultural Deconstruction and Remodeling of the Journey to the West. Movie Review, 5, pp.48-51.

[12] Yuan, K. (2016). Chinese Myths and Legends (in Chinese). Beijing: Beijing United Publishing.

[13] Zhang, J. (2019). On Traditional Culture and Aesthetic Construction in the Animated Film White Snake. Art Evaluation, 24, pp.165-168. 